\title{
La fortezza e il suo giardino: uno sguardo dal mare
}

The fortress and its garden: a view from the sea

\section{Antonio Zunno}

Ministero per i Beni e le Attività Culturali e per il Turismo - Soprintendenza Archeologia Belle Arti e Paesaggio per le Province di Brindisi Lecce Taranto, Lecce, Italy, antonio.zunno@beniculturali.it

\begin{abstract}
The Fortress was built from 1554, on the ruins of an ancient convent, at the behest of Philip of Austria, and it was completed in about 55 years under the direction of Giulio Cesare Falco, knight of the Order of Malta and Captain General against the Turks. The maine structure, called Forte a Mare, was joined with the Opera a Corno, a mighty rampart with the function of enclosure of the intermediate island, separated from the other island in 1598 by the construction of the Angevin canal: here were arranged the lodgings of the troops and garrisons.

Castello and Forte, were named by the Spaniards Isla Fortalera que abre el Puerto Grande, because of its particular position to protect the port. The complex was entrusted to the Germans in 1715, then conquered by the French Revolutionaries and, in 1815, re-annexed to the Kingdom of Naples and destined to lazaretto. A period of decline follows until the end of the 19th century when Brindisi became a first class naval base and the fort became a garrison of the Royal Navy, destined, during the Great War, to recover torpedoes and detonators

The recovery of the complex, starting in the 1980s, allowed the conservation of the structures but was never included in a real valorisation program. With this intervention in progress, a first visit is expected through the visit from the walkways through a circular route from the Castle to the whole Opera in Corno: the itinerary will allow you to retrace the history of the Fortress and enjoy a unique view from the high towards the sea, also through the passage in a curtain of Mediterranean scrub that has colonized the walls over the centuries, creating a veritable hanging garden on the sea. The aim is to lead the visitor to the rediscovery a forgotten place that is closely connected to the coastal landscape, for which it is a privileged point of view also in relation to the city and the port.
\end{abstract}

Keywords: Fortress, sea, rediscovery, landscape.

\section{La storia}

La città di Brindisi ha rivestito sin dall'antichità un'importanza strategica per il territorio a causa della sua naturale insenatura portuale. I Romani infatti elessero il porto di Brindisi quale terminale della via Appia per la comunicazione via mare con il Mediterraneo Orientale. L'isola di Sant'Andrea, situata di fronte all'imboccatura del porto, costitutiva quindi un naturale presidio

per il controllo del traffico di persone e cose e un avamposto di prima difesa. Infatti, sin dal VI secolo d.C. l'isola fu colonizzata da un gruppo di monaci provenieti da Bisanzio che lì fondarono un primo nucleo conventuale fortificato. L'insediamento monastico visse come un'importante e ricca abbazia fino al X secolo quando, dopo l'avvento dei Normanni, in seguito 
alla devastazione saracena, fu insediato sull'isola un nucleo monastico benedettino, destinato a diventare uno dei più grandi insediamenti dell'ordine in Puglia, secondo solo a quello di Taranto. La donazione dell'isola fatta nel 1059 a Melo e Teodomanno da parte di Eustasio, arcivescovo di Brindisi, oltre che per la realizzazione dell'insediamento monastico, farebbe riferimento anche all'erezione di una torre ad defensionem ipsius Sancti Monasterii et savationem anumarum Christianorum. Occorre tuttavia aspettare l'arrivo dei Normanni (1071) perché venga impiantato il cantiere di costruzione dell'abbazia e, conseguentemente, si metta mano alla ipotizzata primitiva opera di difesa, verosimilmente una rudimentale torre in pietra e legno, che doveva fungere anche da faro di segnalazione. Congetturali sono le ipotesi che nel XII secolo l'isola venisse utilizzata con scalo dai crociati e che con Federico II le opere di difesa della città, principale scalo per l'oriente, avessero riguardato anche l'isola di S. Andrea. Allo stesso modo restano da verificare le notizie che Carlo I d'Angiò, creando nuove fortificazioni a difesa del porto, abbia realizzato una torre circolare sull'isola di S. Andrea (1273-1274) e che nel 1410 re Ladislao fece rafforzare il "Castello di mare".

Nel 1445, per volere di Alfonso I d'Aragona si concretizzò il primo intervento finalizzato all'integrazione dell'isola nel contesto difensivo della città, con la costruzione di un massiccio puntone triangolare che inglobò le preesistenti fortificazioni angioine, identificabili in una struttura convessa inglobata nella muratura nord-est e in una struttura circolare scarpate a ridosso del fronte sud-ovest del castello.

Agli inizi del 1481, nell'ambito del generale riassetto del sistema difensivo della costa orientale del regno a seguito della caduta di Costantinopoli per mano di Maometto II (1453), in seguito alla minaccia turca culminata con la presa di Otranto, il sistema difensivo messo a punto dagli Aragonesi determinò la scelta di trasformare Brindisi nel principale presidio militare del Regno. Potenziato il castello detto "di terra", Ferdinando I decise di fortificare l'isola per difendere il porto e la città e fece costruire, inglobando i resti della preesistente struttura angioina, il primo nucleo del futuro castello, affidando al figlio Alfonso II, duca di Calabria, il compito di seguire i lavori. Si deve ad Alfonso I, a seguito della riconquista di Otranto, la trasformazione, a partire dal 1485 del torrione ferdinandeo "riducendolo in vera forma di castello" e accrescendolo in direzione nord-est. A seguito dell'intervento, che vede verosimilmente il coinvolgimento di Francesco di Giorgio Martini, il castello presenta una pianta trapezoidale con ai vertici un bastione triangolare ed un torrione circolare (di S. Filippo, completato sotto il regno di Filippo II, presso l'angolo ovest), impostati su muri a scarpa segnati all'esterno da tori marcapiano e collegati tra loro da un camminamento di guardia. All'interno della struttura militare trovava posto anche la cappelletta a navata unica, dedicata a S. Maria dell'Itria, di cui si conservava l'immagine fino al secolo scorso. La porzione dell'isola che ospitava la fortezza venne isolata attraverso l'escavazione di un canale in corrispondenza del lato est.

Nel 1495, all'interno del forte, oltre che nel castello di terra, i cittadini di parte aragonese trovano scampo all'arrivo delle truppe francesi di Carlo VIII. L'anno successivo, il forte è consegnato ai veneziani insieme alla città.

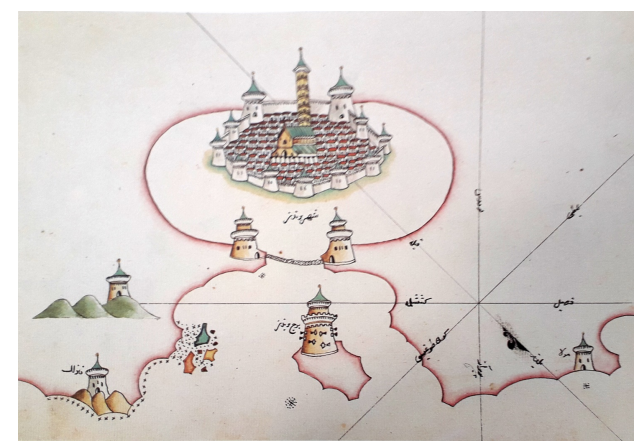

Fig. 1. Il porto di Brindisi nel Kitab -i Bahriye del cartografo turco Piri Re'is (1526).

I turchi ritenevano la città pressochè imprendibile dal mare. L'ammiraglio Piri Reis, nel suo $K i$ tab -i Bahriye scrive, intorno al 1521: "Alla bocca del porto c'è un'isola rocciosa sulla quale è stato costruito un piccolo castello fortificato da cannoni [...] Quest'isola fortificata col piccolo castello si chiama isola di Sant'Andrea". 
Una nuova fase costruttiva, a seguito dell'attacco ad opera di sedici galere venete, comandate da Pietro Lando (1528), ebbe luogo nel XVI, secolo sotto Filippo II, figlio di Carlo $\mathrm{V}$, con il progetto di Antonio Conde e la successiva direzione di alcuni fra i più celebri architetti militari del tempo, fra cui Giulio Cesare Falco, cavaliere dell'Ordine di Malta e più volte Capitano Generale contro i Turchi.

Nel 1557 fu realizzato il "cavaliere" del Castello, ad opera dei maestri di muro brindisini Domenico de Marinis, Mariano de Carlo, Paduano de Balsamo, Giovanni de Parisi, Giorgio Sundo, Evangelista Bruno e Giorgio Lombardo.

Per scongiurare il pericolo di un eventuale assedio di truppe nemiche che, sbarcate sulla parte nord-ovest dell'isola avrebbero potuto piazzare le proprie artiglierie nello spazio vuoto di fronte alla fortezza, nel 1558 si mise mano alla costruzione di un forte (Forte a Mare o Opera a Corno) che, occupando tutto lo spazio disponibile, fungesse da antemurale.

Per consolidare la difesa del complesso, in corrispondenza del lato nord venne realizzato il canale "Angioino", con le sponde foderate di muratura e attraversato da un ponte in muratura, completato nel 1598 con l'intento di isolare completamente il forte dalla restante parte dell'isola di S. Andrea sulla quale fu costruito un altro grande antemurale, ulteriore barriera per l'avvicinamento dei potenziali nemici al forte.

Il complesso delle fortificazioni sull'isola di S. Andrea, completato nel 1609, assume l'aspetto che conserverà per secoli; l'immagine che si ricava dalla cartografia storica è quella di un possente vascello a guardia e protezione della città, cui rivolge la prua, corrispondente all'emergenza, anche visiva, del castello alfonsino, la cui mole si innalza sensibilmente rispetto alla retrostante opera a corno.

Sulla parte dell'isola non occupata da strutture militari venne costruito, nei primi del Settecento per volontà di Carlo III di Borbone, un lazzaretto, in uso sino ai primi anni del XIX secolo. A seguito dei trattati di Utrecht e di Radstadt, il complesso fu affidato nel 1715 agli austriaci.
Il complesso venne espugnato e conquistato dai Rivoluzionari francesi e riannesso, nel 1815, al patrimonio del Regno di Napoli e destinato a lazzaretto.

Nel 1868 l'Isola di S. Andrea viene collegata alla terraferma tramite un lungo ponte in muratura, denominato "Bocche di Puglia".

Risale al 1843 l'installazione sulla sommità della rocca alfonsina, sul sito dove era stato in precedenza stabilito il telegrafo, di un faro di segnalamento "di VI classe" a servizio della navigazione, con lanterna composta da 8 lumi.

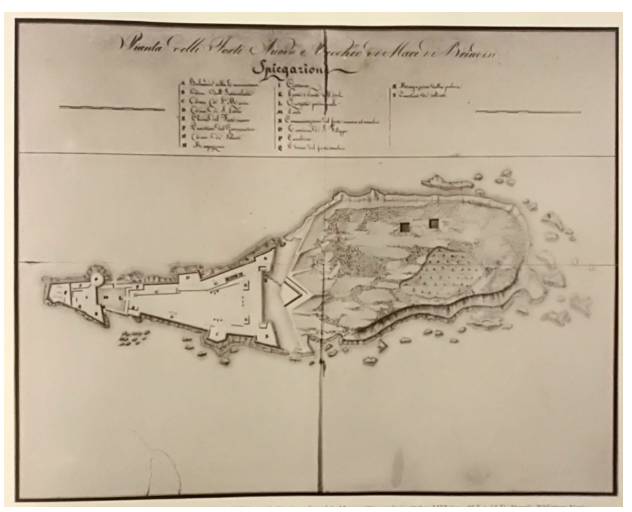

Fig. 2. Pianta delli Forti nuovo e vecchio di mare di Brindisi, ottobre 1817 (49,7 x 63,7 cm). Napoli, Biblioteca Nazionale "Vittorio Emanuele III", B. 5C/20.

La situazione di sotto-utilizzazione del complesso, descritta dal Castromediano nel 1848 si protrae per tutto il XIX secolo; gli spazi del forte vengono utilizzati in maniera varia e discontinua ed alcuni ambienti sono adibiti a carcere militare di rigore. Dopo alcuni anni viene tolto il presidio militare ancora presente nella Rocca Alfonsina. Nel 1886-1887 il Consiglio Comunale di Brindisi chiede al Ministero dell'Interno la concessione di alcuni locali del Forte per impiantarvi una lavanderia per le biancherie per la prevenzione del colera, a seguito della rinnovata importanza dello scalo portuale conseguente all'apertura del canale di Suez (1869). Tra la fine del XIX e gli inizi del XX secolo vengono effettuate alcune operazioni di manutenzione delle strutture difensive, a cura della Marina Militare, del Genio Militare di Bari e Taranto e del Genio Civile. 
Dopo il lungo periodo di declino, allo scoppio della Prima Guerra Mondiale, Brindisi diviene nel 1911 una base navale di primo piano ed il Forte a Mare diviene presidio della Regia Marina, che restaura e potenzia le fortificazioni esistenti, creando nuovi depositi per le torpedini ed i relativi detonatori, postazioni fisse di artiglieria ed alloggi all'interno dell'Opera a Corno.

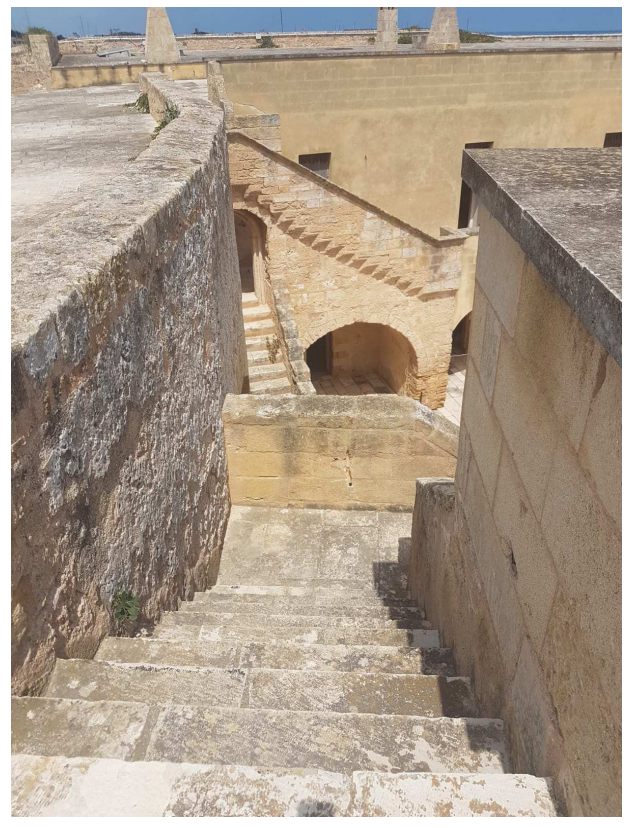

Fig. 3. Scalinate di raccordo tra i camminamenti di ronda, gli alloggi dei militari situati nelle maniche dell'Opera a Corno e la piazza d'armi.

Dalla metà del secolo scorso la struttura affronta un nuovo periodo di lento declino, anche a seguito del graduale abbandono del presidio da parte dei militari; negli anni '70 lo stato di incuria determina una serie di crolli. Fino al 1980 la struttura ospita la sede amministrativa del Comando Marina di Brindisi, ma nel 1982 il Comando Marina trasferisce gli ultimi uffici ancora attivi ed alla fine dell'anno successivo si assiste alla dismissione del complesso, preso in consegna dal Demanio dello Stato, che lo affida nel 2000 alla Soprintendenza per i Beni Ambientali, Architettonici, Artistici e Storici della Puglia che interviene dando inizio ai lavori di recupero e valorizzazione.

\section{Il progetto}

L'intervento appena concluso è il primo stralcio di un percorso di valorizzazione che si prefigge di ridare una nuova vita al complesso monumentale. L'obiettivo è di garantire permanentemente l'apertura al pubblico, attraverso un percorso di valorizzazione che, oltre ad evidenziare le caratteristiche proprie dell'unicità del sito, coniughi anche l'esigenza di nuovi spazi da offrire alla città di Brindisi, affinché la città stessa possa riappropriarsi di un bene fortemente identitario. Oltre alle necessarie operazioni di restauro delle cortine murarie che versavano incondizioni di precaria stabilità e conservazione, a causa della peculiarità del sito praticamente circondato dal mare, l'intervento ha dovuto far fronte anche al recupero dei vani già oggetto di lavori realizzati negli anni scorsi ma brutalmente vandalizzati. $\mathrm{Al}$ fine di realizzare i servizi minimi di accoglienza e utilizzo dell'immobile, sono stati ripristinati i locali della guardiania, posti immediatamente all'ingresso all'Opera a Corno dal sottarco che conduce alla darsena interna e l'ampia sala dell'ex Chiesa, adibita a punto di orientamento alla visita e ad installazioni temporanee. Nuovi impianti, locali igienici e completamento delle finiture permetteranno di garantire un presidio permanente della stuttura.

La grande sfida però, in questa fase, è stata la realizzazione un percorso di visita e conoscenza del complesso fortilizio attraverso un itinerario, quasi tutto in esterno, che potesse accompagnare il visitatore alla scoperta di un singolare contesto dall'alto valore paesaggistico. All'interno dell'Opera a Corno infatti, nei secoli si è generato un micro mondo verde che ha colonizzato il cavaliere a nord fino ad invadere l'intera cortina muraria che affaccia sul canale Angioino. Attraverso un'operazione di decespugliamento selettivo è stato riportato alla luce l'antico selciato del tratto di camminamento di ronda del muro nord che ha permesso quindi di definire un più ampio percorso di visita dedicato alla muraglia. E' stato ritrovato anche un innesto murario risalente al primo conflitto modiale che è stato recuperato alla stregua degli altri elementi caratteristici dell'architettura militare, rinvenuti sotto la coltre vegetale. 
Il percorso di visita è stato ideato come un percorso paesaggistico che attraverso i camminamenti di ronda dell'Opera a Corno permette di immergersi anche nel delizioso giardino pensile di macchia mediterranea, cresciuto isolato dalla terra ferma sul cavaliere interno, sopra le cisterne seminterrate nella parte nord dalla piazza d'armi.

L'intento del percorso paesaggistico è permettere di osservare la città da un punto divista privilegiato: essendo l'isola rivolta verso all'imboccatura del porto, è possibile dialogare con la vista della città dal mare e godere dell'orizzonte verso il mare aperto da una prospettiva inedita.

Per garantire la continuità del percorso in quota, è stato necessario ripristinare il collegamento del camminamento di ronda del muro ad est, nel tratto che congiungeva la fortificazione dell'Opera a Corno con il Forte a Mare attrevarso la darsena. Il rovinoso crollo del muro est, in questa fase dell'intervento indagato anche dal punto divista archeologico, fu determinato con molta probabilità da una mutata stabilità del nucleo a causa della continua esposizione a i marosi e alla consistenza mutevole del terreno su cui sorgeva, caratterizzato da scogli artificiali addossati ad un lembo di terreno limoso. Il ripristino del perscorso del camminamento di ronda avviene quindi mediante la ricostruzione del tratto di muratura perduto con una struttura in legno, munita di appoggi puntuali e distribuiti lungo la base del vecchio muro, che collega il tratto residuo che congiunge alla Porta Regia del primo piano del Forte a Mare. Durante i restauri della muratura sommitale di tale tratto è stato possibile recuperaree l'originale conformazione del parapetto, caratterizzata da conci in pietra sagomati a scarpa verso l'esterno, precedentmente inglobati da una murarura sovrapposta successivamente.

L'eliminazione dei filari di muratura aggiunta ha permesso di alleggerire la lettura del ponte in pietra, su cui si inesta anche una garitta di guardia (al cui interno sono stati ritrovati alcuni graffiti raffigurati un castello e un veliero), e valorizzare il distacco del cammino di ronda dall'accesso alla Porta Regia, che in origine avveniva per mezzo di un ponte levatoio.

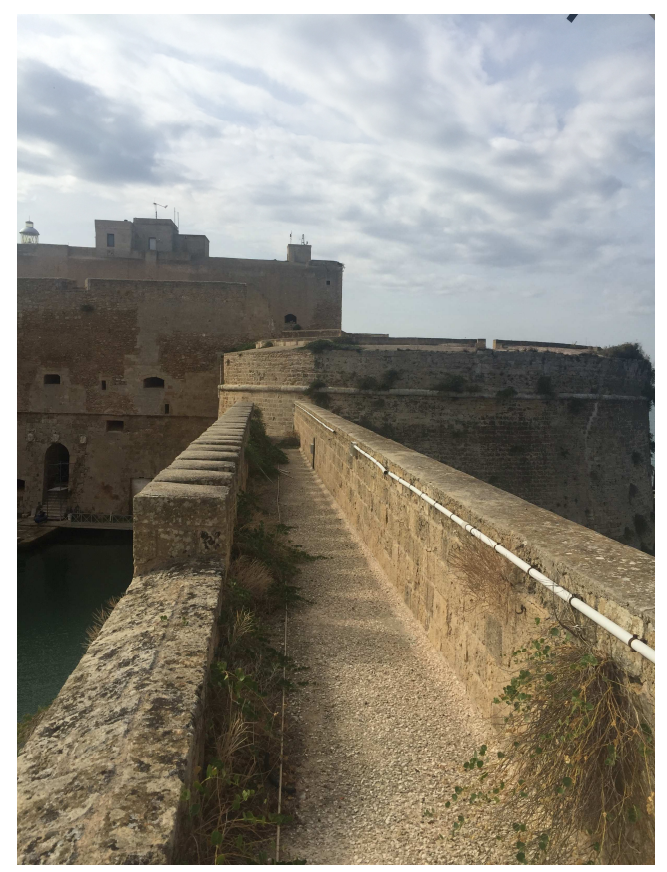

Fig. 4. Parte del camminamento di ronda che connetteva il bastione angioino.

La messa in sicurezza di tutto il percorso in quota permette di raggiungere l'interno del forte da un ingresso privilegiato e accessibile anche ai portatori di disabilità motoria: attraverso un percorso dedicato, recuperata anche la rampa in muratura nella piazza d'armi, gli utenti affetti da limitazioni motorie potranno raggiungere la quota del camminamento di ronda dal lato nord dell'Opera a Corno e precorrere il suggestivo itinerario verso il castello, raggiungendo direttamente la terrazza a livello del primo piano. Da qui l'ingresso alle sale del primo piano permette la visita al piano nobile del forte, caratterizzato da un ampio salone voltato, impreziosito da un caminetto con ormento scolpito e da un catino in pietra incastonato dentro una cornice in pietra lavorata. Gli interni delle sale, ormai privi di finiture, potranno accogliere nuovi usi, dai convegni alle mostre ad altre iniziative compatibili.

Al piano terra del forte, attraverso il recupero di un passaggio precedentemente obliterato da un 
muro, si potrà accedere ai vani che si trovano alla base del castello, altissimi e coperti a volta, che hanno ospitato durante i conflitti mondiali il ricovero delle artiglierie e delle torpedini.

Nella piazza d'armi dell'Opera a Corno, è stata allestita un'area di circa $400 \mathrm{~m}^{2}$ mediante il recupero e ricollocamento in opera dell'antico basolato divelto dai camminamenti in quota, creando uno spazio utile alla realizzazione di piccoli eventi. Tra le due maniche degli alloggi dei militari, che cingono la piazza d'armi, è stata riservata invece un'area libera adatta a grandi eventi o manifestazioni pubbliche: le condizioni di fruizione dei percorsi sono state ristabilite mediante la creazione di due direttrici realizzate in terra stabilizzata e finitura ecocompatibile che permettono anche il ricongiungimento ai percorsi paesaggistici in quota attraverso la rampa in muratura.

Il percorso naturalistico all'interno dell'area verde è dedicato alla scoperta delle piante tipiche della macchia mediaterranea, con aree di sosta e percorsi accessibili muniti di comunicazione dedicata anche ad ipovedenti.

Tutto il percorso di visita è accompagnato da una guida virtuale con l'ausilio di una app che attraverso 22 points of interest racconta la storia dei luoghi, descrive il rapporto tra il complesso e il mare, il sito e la città con il suo porto, le scoperte archeologiche rinvenute durante i lavori. Il percorso di visita è anche arrichito da una sala immersiva, situata all'interno di uno dei vani del piano primo del castello, dove un racconto virtuale ripercorre le fasi dell'assedio dei veneziani attraverso la voce narrante di uno dei protagonisti dell'evento.

\section{Conclusioni}

Le istanze collettive relative alla richiesta di fruizione dei monumenti necessitano di una riflessione sull'approccio alla valorizzazione declinata secondo i tempi moderni.

Di sicuro, le mutate modalità di veicolazione delle informazioni e la possibilità di reperire le stesse in rete, impongono che la comunicazione di un sito debba necessariamente riferirsi alla costante richiesta di novità.

Per quanto il tema della conservazione sia ormai parte del comune sentire civico, esistono contesti dove la mancanza di informazione corretta circa l'unicità di un sito culturale, porta a sottovalutare il valore di un recupero da parte popolazione. Per quanto la città di Brindisi sia da sempre attratta dal complesso del Forte a Mare, a causa

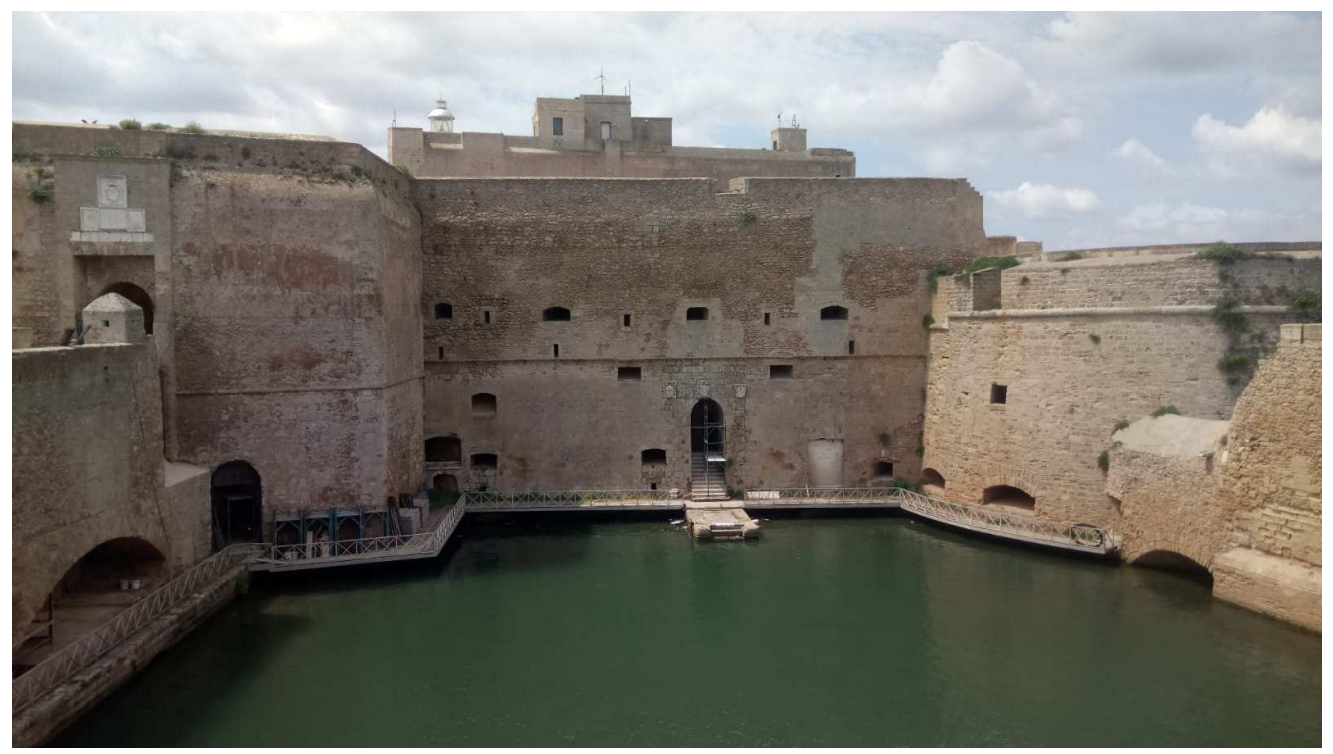

Fig. 5. Il Forte visto dai camminamenti di ronda dell'Opera a Corno. 


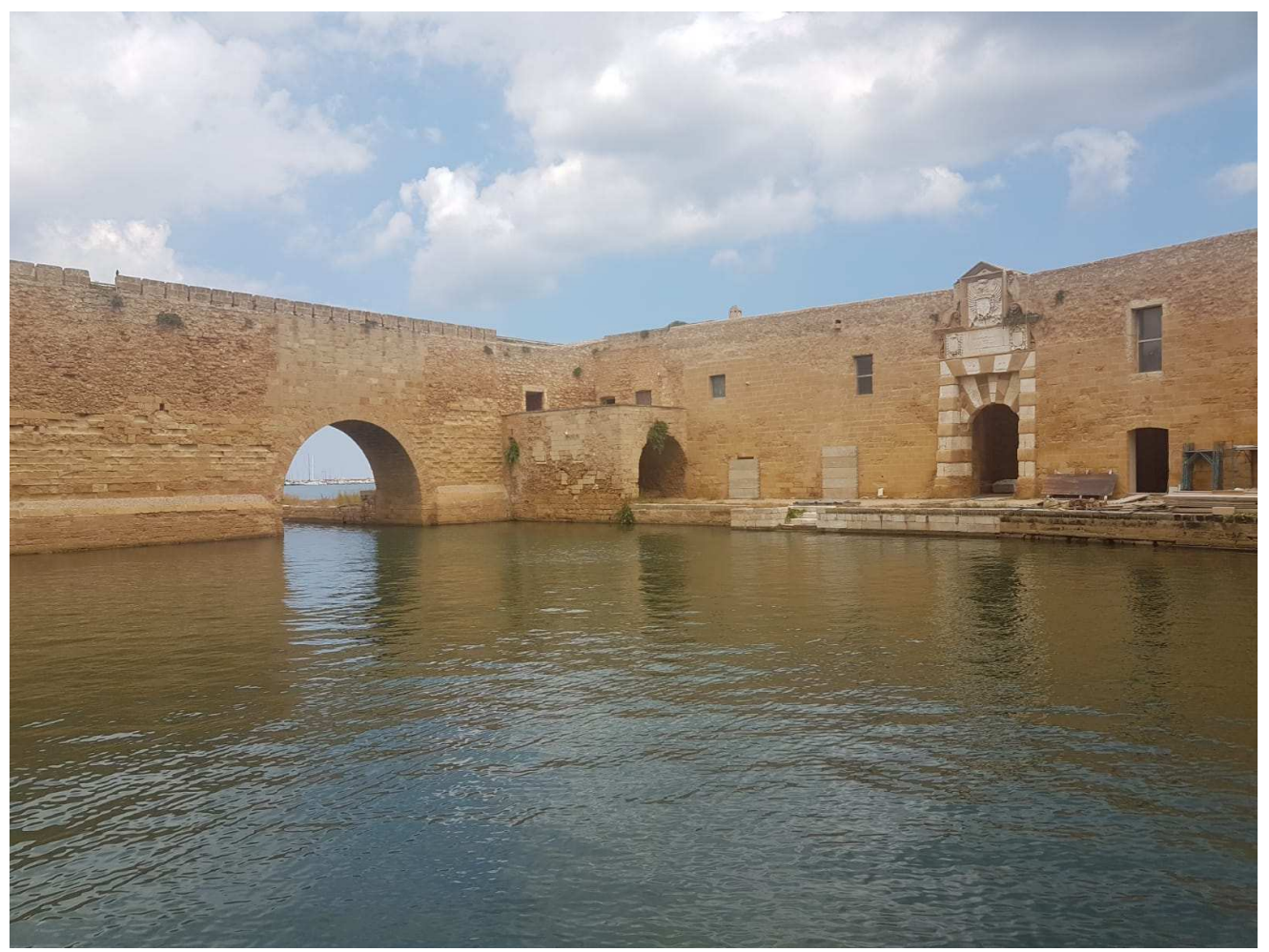

Fig. 6. La porta Alfonsina dell'Opera a Corno vista dal Forte a Mare: ingress alla darsena dal porto turistico.

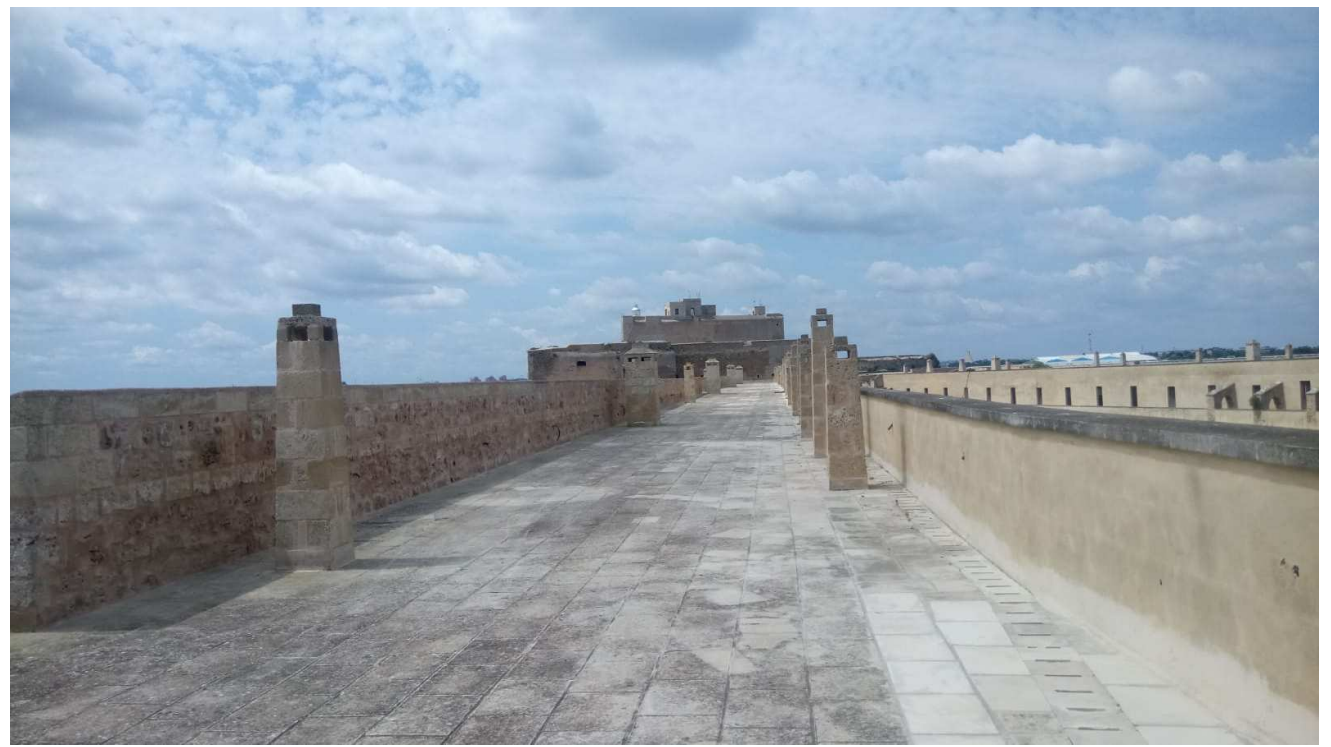

Fig. 7. Il camminamento di ronda della cortina Est dell'Opera a Corno: camini di sfiato degli ex alloggi military. 
della continua presenza dei militari, da sempre l'accesso al sito è stato di fatto impedito. I primi lavori di recupero sono stati vanificati a causa degli episodi vandalici operati di certo in assenza della dovuta informazione circa il destino del monumento.

In questa fase, invece, una serie di aperture straordinarie rivolte all'amministrazione e a gruppi organizzati, ha permesso la conoscenza del cantiere, delle varie fasi dell'intervento e del- le finalità del recupero, destando molto interesse e ingenerato aspettative importanti.

La riapertura al pubblico, l'organizzazione di visite guidate ed eventi culturali sarà accolta con spirito di rinnovata appartenenza proprio perchè tutta questa operazione è stata comunicata e condivisa nel tempo.

\section{Bibliography}

Amirante, G.; Pessolano, M.F. (2005). Immagini di Napoli e del Regno: le raccolte di Francesco Cassiano de Silva, Napoli - Roma.

D'Armento, F. (2017). L'Abadia di S. Andrea dell'Isola di Brindisi e i suoi feudi salentini: Brindisi, Campo Longobardo, Latiano, S. Giovanni Monicantonio, Campie Misciagne: trascrizione di un manoscritto del 1627 con analisi storica, critica, paleografica e appendice documentaria, Lecce.

Briamo, F.; Cavaliere. G. (1972). Brindisi: storia scritta da secoli di miseria e di morte: il canale Pigonati, Galatina.

Brunetti, O. (2006). L'ingegno delle mura: l'Atlante Lemos della Bibliothàeque Nationale de France, Firenze.

Camassa, P.; Brindisi, G. di. (1897). Ristampa anastatica, Brindisi, Lecce.

Carlone, G.; Angelini G. eds. (1996). Castelli e fortificazioni in Puglia: visite alle difese marittime nell'età del viceregno spagnolo, Manduria.

Casanova, L. (1996). "Il castello rosso a Brindisi: dall'abbandono a museo attivo degli sport del mare", Continuità. Rassegna Tecnica Pugliese, 3-4, pp. 98-108.

Coco, P.A. (1919). L'abadia di S. Andrea dell'Isola in Brindisi: studio storico critico con documenti inediti, Lecce.

Cuciniello, D.; Bianchi, L. (1971). Viaggio pittorico nel Regno delle Due Sicilie, Napoli.

Vita, R. De., ed. (1974). Castelli, torri ed opere fortificate di Puglia, Bari.

VV.AA. (1986). Forte a Mare. Simbolo per il recupero architettonico del Salento, Galatina.

VV.AA. (1998). Il castello, la marina, la città: mostra documentaria, Galatina. 